\title{
Variación temporal de la distribución espacial por edades de Phoenicopterus ruber (Phoenicopteriformes: Phoenicopteridae) en los humedales de Yucatán, México
}

\author{
Alexis Herminio Plasencia-Vázquez ${ }^{1}$, Xiomara Gálvez-Aguilera ${ }^{2}$, Yarelys Ferrer-Sánchez ${ }^{3}$ \& \\ Anay Serrano-Rodríguez ${ }^{4}$ \\ 1. Centro de Investigaciones Históricas y Sociales (CIHS), Universidad Autónoma de Campeche, Av. Agustín Melgar, \\ s/n, CP 24030, Campeche, Campeche; alexpla79@gmail.com \\ 2. Niños y Crías, A.C., Programa Integral de Conservación del Flamenco del Caribe, Mérida, México; \\ xiogalveza@yahoo.com.mx \\ 3. Facultad de Ciencias Ambientales, Universidad Técnica Estatal de Quevedo, Quevedo, Ecuador; \\ yferrersanchez@gmail.com \\ 4. El Colegio de la Frontera Sur (ECOSUR), Unidad Campeche. Av. Rancho Polígono 2-A, Ciudad Industrial de Lerma, \\ C.P. 24500, Lerma, Campeche, Mexico; anayserrano1984@gmail.com
}

Recibido 13-II-2017. Corregido 10-VII-2017. Aceptado 09-VIII-2017.

\begin{abstract}
Temporal variation in spatial distribution by age of Phoenicopterus ruber (Phoenicopteriformes: Phoenicopteridae) in the wetlands of Yucatán, Mexico. The Caribbean Flamingo (Phoenicopterus ruber) in Mexico is distributed throughout the complex of lagoons in coastal wetlands of the Yucatan Peninsula. The species shows a tendency toward differential use of areas between juveniles and adults at different stages of their life cycle, and in different seasons. The aim of this study was to determine the most important areas where flamingos are distributed in Yucatan wetlands, and to describe temporal variations according to age and stages of their life cycle. For this, we used the records of ringed individuals sighted during the period 2010-2015. The areas with the largest numbers of individuals, by stage and season, were identified. Flamingos were recorded in 39 different areas of 43 sampled. In general, the sites with the largest number of records were Isla La Angostura and Punta Mecoh. To both juveniles and adults, areas with higher occupancy rates differed by age between the different stages of the life cycle and seasons. In the Charca Salinera Chel and Isla La Angostura ringed flamingos of almost all ages were recorded. Although already described areas (Celestún, Ría Lagartos) are certainly important, new sites hosting considerable populations at some stage of this species life cycle were detected (e.g. Humedal Progreso; Salamandra, Laguna Rosada; Xcambo). These new sites should be given more attention and important security measures and protection are recommended to all of them. It is necessary to seek new support sources for studies that determine the flamingo population's status in difficult access areas for which little information is available. Rev. Biol. Trop. 65 (4): 1483-1495. Epub 2017 December 01.
\end{abstract}

Key words: adults, Caribbean Flamingo, endangered species, juveniles, Phoenicopteridae, Yucatan Peninsula.

En la actualidad, los factores naturales se conjugan con los antrópicos y en su conjunto determinan las áreas que las especies ocupan (Plasencia, Escalona, \& Esparza, 2014). Delimitar la distribución resulta más sencillo cuando hay barreras geográficas evidentes, pero resulta una tarea compleja en zonas donde no se producen cambios abruptos en las variables abióticas y bióticas (Cueto, Milesi, Sagario,
López, \& Marone, 2011). En el caso de las aves, se ha comprobado en algunas especies, que las diferentes clases de edades y de sexos a menudo ocupan diferentes hábitats, lo cual se ha relacionado con la exclusión competitiva y con las diferencias en la calidad del hábitat (Pérez \& Telleria, 2002; Latta \& Faaborg, 2002). En algunos casos, estas diferencias pueden estar dadas por un uso diferencial 
de las zonas de alimentación por dominancia intraespecífica (Donázar, Travaini, \& Ceballos, 1999). Los juveniles tienden a diferir de los adultos conespecíficos en su dieta y en los métodos de captura y manipulación de las presas (Marchetti \& Price, 1989). Estas diferencias están dadas por la inmadurez del pico y del sistema esqueleto-muscular y neurológico, así como por el tiempo necesario para aprender habilidades de búsqueda de alimento (Marchetti \& Price, 1989).

El flamenco caribeño (Phoenicopterus ruber) es un ave colonial, que habita en las lagunas salinas, lagos interiores, marismas y costas salobres poco profundas, donde forrajea y se reproduce en enormes bandos de hasta 24000 individuos, conformados por diferentes grupos etarios (Migoya \& Tabasco, 2010). Actualmente en México, el flamenco caribeño se distribuye en todo el complejo de lagunas dentro de los humedales costeros de la Península de Yucatán, desde la Reserva de la Biosfera Los Petenes en el estado de Campeche hasta la Isla Holbox en Quintana Roo, aunque hay algunos registros ocasionales en la Reserva de la Biósfera Sian Ka'an y en la Isla de Cozumel (Gálvez, Gavaldón, Cruz, Guerrero, \& Marvi, 2014). El sitio fundamental para anidar continua siendo Ría Lagartos en el estado de Yucatán. Fuera de la Península de Yucatán, en territorio mexicano los reportes de la especie son escasos y el registro más distante data de 2013 en la costa del estado de Nayarit, donde se observaron dos ejemplares que pudieron haber escapado del cautiverio (Mendoza, Cortés, Sierra, \& Carmona, 2013).

Entre las principales amenazas para los flamencos están la pérdida de hábitat, ocasionada por la degradación de las zonas costeras debido al desarrollo urbano y turístico (Galicia \& Baldassarre, 1997). A largo plazo, esta situación pudiera agravarse debido al ascenso en el nivel del mar producto del calentamiento global (Andrade, 2010). Los flamencos también se ven afectados por los contaminantes que llegan a las áreas donde se desarrollan, provocándoles enfermedades (Thomas, Hunter, \& Atkinson, 2008). Además, son sensibles al envenenamiento por plomo en las zonas utilizadas para la cacería de patos (Schmitz, Alonso, Cook, \& Bald, 1990), y por medicamentos, pesticidas y otros productos químicos que contaminan los sistemas acuíferos (Escalante, 2010). También sufren lesiones y muerte por colisión con tendidos eléctricos.

Durante las observaciones realizadas de la especie, como parte del Programa Integral de Conservación del Flamenco en Yucatán, México, se ha observado una cierta tendencia a un uso diferencial de las áreas entre los juveniles y adultos en las diferentes etapas de su ciclo de vida y durante las variadas estaciones del año. Para poder corroborar esta hipótesis, se definió como el objetivo de este estudio, determinar las áreas más importantes donde se distribuyen espacialmente los flamencos en los humedales de Yucatán, México, y sus variaciones temporales según la edad y las diferentes etapas de su ciclo de vida, a partir de registros de individuos anillados que se han avistado durante el período 2010-2015. De esta forma, se puede contribuir de manera más concreta, con acciones de manejo del hábitat de la especie en aquellos hábitats críticos durante diferentes etapas de su ciclo de vida.

\section{MATERIAL Y MÉTODOS}

Área de estudio: El Estado de Yucatán está situado en el extremo norte de la Península de Yucatán $\left(19^{\circ} 32^{\prime}\right.$ - $21^{\circ} 36^{\prime}$ N \& 87³2' - 90²5' W), en el sureste de la República Mexicana. Colinda al norte con el Golfo de México, al este y sureste con el estado de Quintana Roo y al oeste y suroeste con el estado de Campeche (CONABIO, 2006). Se presentan tres épocas climáticas a lo largo del año: secas de febrero a mayo, lluvias de junio a septiembre y nortes de octubre a marzo (García, 2004).

Datos de la especie: Se procesaron los datos de individuos anillados del flamenco caribeño en el estado de Yucatán, Península de Yucatán, que se registraron durante el intervalo 2010-2015. Los individuos fueron anillados en el período 1996-2014, como parte del Programa 
Integral de Conservación del Flamenco, liderado por la Comisión Nacional de Áreas Protegidas (CONANP) y la Organización Niños y Crías A. C. Se muestrearon 43 áreas en las que se tenía certeza de la presencia de la especie en la región, como resultado de los 13 años de censos aéreos anuales realizados, a partir de los cuales se documentaron los sitios de descanso, reproducción y alimentación de flamencos en la Península de Yucatán (Gálvez et al., 2014). En cada una de estas áreas se establecieron puntos de observación estratégicos, que permitieran una mejor visibilidad de la colonia, sin alterar la misma. En cada punto, con la ayuda de binoculares, se rastrearon hacia el interior de la colonia los individuos que estuvieran anillados y se tomaron las numeraciones y códigos de los mismos. Con el dato del año de anillamiento y del registro, se calculó la edad que tenía cada flamenco cuando fue avistado. Se clasificaron a los individuos entre 0-5 años en la categoría de juveniles y los mayores de cinco años como adultos. Además, se clasificaron los registros, teniendo en cuenta si habían sido tomados dentro o fuera de las etapas pre-reproductiva (Pre-R), Reproductiva (R) y pos-reproductiva (Pos-R) de la especie. Estas etapas son diferentes entre adultos y juveniles. Para lo juveniles se estableció que: la etapa Pre-R (enero-marzo), R (abril-agosto) y Pos-R (septiembre-diciembre), mientras que para los adultos: Pre-R (noviembre-marzo), R (abrilagosto) y Pos-R (septiembre-octubre). También se tuvieron en cuenta las tres estaciones del año propuestas por García (2004) para la región.

Manejo de datos: Se determinó el número total de individuos registrados y el número total de avistamientos según su edad y el total de áreas visitadas, durante el período 20102015. El número de avistamientos está dado porque un mismo individuo se pudo registrar en diferentes momentos del año o en diferentes áreas. Para el número total de individuos y de avistamientos, se determinaron los estadísticos básicos (media, desviación estándar y mínimo-máximo). Se identificaron las áreas con el mayor porcentaje de individuos durante el intervalo 2010-2015, en función de la edad y las etapas Pre-R, R y Pos-R, en las diferentes estaciones del año.

\section{RESULTADOS}

De manera general, se registraron flamencos entre 2010-2015 en 39 áreas diferentes, de 43 muestreadas (Fig. 1, Cuadro 1). En la Charca Salinera Chel y en Isla La Angostura fueron en las que se registraron flamencos anillados de casi todas las edades, excepto los más longevos de 19 años (Cuadro 1). En las áreas de reproducción Isla La Angostura y Punta Mecoh se observó el mayor número de individuos anillados (2008 y 1336 respectivamente) y le continuaron varias de las Charcas Salineras aledañas (Cuadro 1). Fuera de las áreas de reproducción o colindantes a estas, las áreas con los mayores registros fueron Isla Pájaros, Celestún; Pilares, Celestún y la Ría Natural frente a Yoluk (Cuadro 1). Existen otras áreas en las cuales se registraron muy pocos individuos anillados de flamencos, como es el caso de Laguna Champolak y Laguna Nahular, ambas en Sisal o Chuburná; por lo cual no sobresalieron entre las más importantes (Cuadro 1).

El número total de áreas en que se localizaron los flamencos fue variable, y a partir de los 16 años fue menor el número de sitios en que se registraron (Cuadro 1, Cuadro 2). El número total de registros $\mathrm{y}$ de avistamientos del flamenco caribeño fluctuó entre individuos de 0-19 años de edad (Cuadro 2). Los mayores valores se obtuvieron para los flamencos de 0 , 10 y 11 años (Cuadro 2).

En el caso de los juveniles, las áreas con mayor número de individuos anillados fueron diferentes por edades entre las diferentes etapas del ciclo de vida y las estaciones del año (Fig. 2A). De los individuos de 0 años solo se tuvo información durante la etapa Pos-R-LL y Pos-R-N. En un inicio, los pollos anillados se concentraron en la Charca Salinera la Esperanza y en Isla La Angostura, y ya durante la época de nortes, se movieron en su mayoría a Isla Pájaros, Celestún y el Parador Dzinitún en Celestún, aunque una parte considerable 


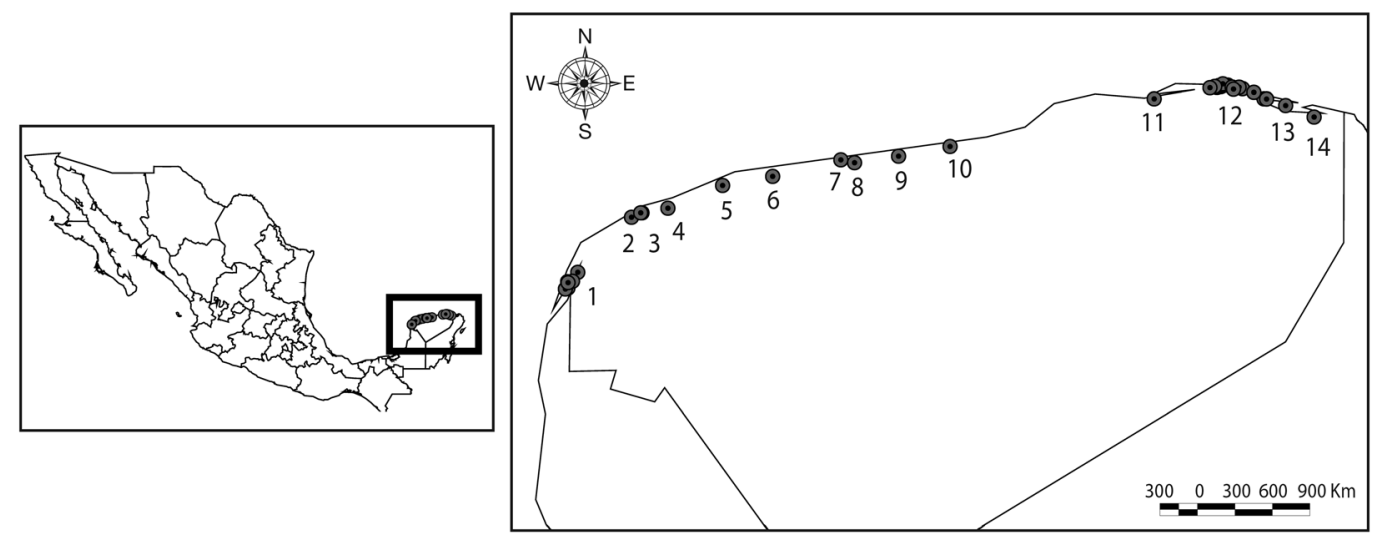

Fig. 1. Áreas en las que se registraron individuos anillados de flamenco caribeño (Phoenicopterus ruber) durante el período 2010-2015 en Yucatán, México (dentro del rectángulo). 1. Incluye: Charca Salinera Podridos; Isla Pájaros, Celestún; Laguna cerca Puerto Abrigo Celestún; Parador Dzinitún; Pilares, Celestún y Charca Salinera Plaza de Armas, Celestún. 2. Laguna Champolak-Sisal. 3. Incluye: Laguna Baas, Sisal y Laguna Nahular, Sisal. 4. Humedal carretera Sisal-Hunucmá. 5. Chuburná. 6. Humedal Progreso. 7. Salamandra, Laguna Rosada. 8. Xcambo. 9. Charca San Crisanto. 10. Mina de Oro. 11. Fraccionamiento Fox, San Felipe. 12. Incluye: Bomba Casa del Rio; Mulsunik, Ría Lagartos; Ria Natural frente Yoluk; Angostura; Isla La Angostura; Punta Mecoh; Charcas Salineras: Anteojo Morano, Becamay, Chel, Chocolatera, Chojon, Cruz Grande, Miguel Torres, Poceb Xac, Polkos, Salines, Vía 3, Vía Oriente, La Esperanza y San Fernando. 13. Alegrías, Ría Lagartos. 14. Punta Manolo, El Cuyo.

Fig. 1. Areas where ringed individuals of Caribbean Flamingo (Phoenicopterus ruber) were recorded during the period 2010-2015 in Yucatan, Mexico (inside the box). 1. Include: Charca Salinera Podridos; Isla Pájaros, Celestún; Laguna cerca Puerto Abrigo Celestún; Parador Dzinitún; Pilares, Celestún y Charca Salinera Plaza de Armas, Celestún. 2. Laguna Champolak-Sisal. 3. Include: Laguna Baas, Sisal y Laguna Nahular, Sisal. 4. Humedal carretera Sisal-Hunucmá. 5. Chuburná. 6. Humedal Progreso. 7. Salamandra, Laguna Rosada. 8. Xcambo. 9. Charca San Crisanto. 10. Mina de Oro. 11. Fraccionamiento Fox, San Felipe. 12. Include: Bomba Casa del Rio; Mulsunik, Ría Lagartos; Ria Natural frente Yoluk; Angostura; Isla La Angostura; Punta Mecoh; Charcas Salineras: Anteojo Morano, Becamay, Chel, Chocolatera, Chojon, Cruz Grande, Miguel Torres, Poceb Xac, Polkos, Salines, Vía 3, Vía Oriente, La Esperanza y San Fernando. 13. Alegrías, Ría Lagartos. 14. Punta Manolo, El Cuyo.

se mantuvo cerca de las áreas de reproducción en la Charca Salinera Salines (Fig. 2A).

Durante la etapa Pre-R-N, los individuos anillados de 1 año de edad se concentraron en su totalidad en Isla Pájaros, Celestún; mientras que entre 2 y 5 años de edad, en general, la Charca Salinera Chel figuró entre las más importantes (Fig. 2A). En la etapa Pre-R-S los jóvenes de 1 año se dispersaron en un mayor número de áreas respecto a los de 2 a 5 años, que se mantuvieron en su mayoría en la Charca Salinera Chojon (Fig. 2A). Durante el inicio de la reproducción, en R-S, los individuos de 1 año se mantuvieron de igual manera más dispersos, mientras que los de 2 a 5 años se concentraron más entre Isla La Angostura y Punta Mecoh (Fig. 2A). En la etapa R-LL se mantuvo el mismo patrón que al inicio de la reproducción en secas, los de 1 año continuaron más dispersos y de 2 a 5 años se concentraron más en Isla La Angostura, la Charca Salinera Chel y Punta Mecoh (Fig. 2A). Ya durante la etapa Pos-RLL, se comportaron de forma similar desde 2 a 5 años de edad, y se ubicaron en su mayoría en algunas de las Charcas Salineras y en Isla La Angostura. En Pos-R-N se movieron una gran parte a Isla Pájaros, Celestún; mientras que otro grupo considerable se mantuvo mayormente en la Charca Salinera Chel (Fig. 2A).

En el caso de los adultos, también se observaron variaciones entre las áreas con mayor cantidad de individuos durante las diferentes etapas del ciclo de vida y las estaciones del año (Fig. 2B). En Pre-R-N, de manera general, las áreas más importantes por el número de individuos que presentaron fueron la Charca Salinera 


\section{CUADRO 1}

Áreas en las que se registraron individuos anillados de flamenco caribeño (Phoenicopterus ruber) durante el período 2010-2015 en Yucatán, México

TABLE 1

Areas where ringed individuals of Caribbean Flamingo (Phoenicopterus ruber) were recorded during the period 2010-2015 in Yucatan, Mexico

\begin{tabular}{|c|c|c|c|c|c|}
\hline ID & Sitios & Total Individuos & Latitud & Longitud & Edades (años) \\
\hline 1 & Alegrías, Ría Lagartos & 1 & $21^{\circ} 31^{\prime} 32.94 " \mathrm{~N}$ & $87^{\circ} 45^{\prime} 14.97^{\prime \prime} \mathrm{O}$ & 9 \\
\hline 2 & Angostura & 58 & $21^{\circ} 32^{\prime} 59.97^{\prime \prime} \mathrm{N}$ & $87^{\circ} 50^{\prime} 0.06^{\prime \prime} \mathrm{O}$ & $0,2-12,15-16$ \\
\hline 3 & Bomba Casa del Rio & 26 & $21^{\circ} 35^{\prime} 43.97^{\prime \prime} \mathrm{N}$ & $87^{\circ} 59^{\prime} 43.97^{\prime \prime} \mathrm{O}$ & $0-2,5-7,9-13$ \\
\hline 4 & Charca Salinera Anteojo Morano & 1 & $21^{\circ} 35^{\prime} 41.83^{\prime \prime} \mathrm{N}$ & $87^{\circ} 59^{\prime} 30.98^{\prime \prime} \mathrm{O}$ & 4 \\
\hline 5 & Charca Salinera Becamay & 99 & $21^{\circ} 35^{\prime} 30.46^{\prime \prime} \mathrm{N}$ & $88^{\circ} 0 ’ 21.00^{\prime \prime} \mathrm{O}$ & $0-1,3-12$ \\
\hline 6 & Charca Salinera Chel & 588 & $21^{\circ} 35^{\prime} 59.46^{\prime \prime} \mathrm{N}$ & $87^{\circ} 57^{\prime} 57.91^{\prime \prime} \mathrm{O}$ & $0-18$ \\
\hline 7 & Charca Salinera Chocolatera & 135 & $21^{\circ} 35^{\prime} 15.05^{\prime \prime} \mathrm{N}$ & $87^{\circ} 54^{\prime} 41.57^{\prime \prime} \mathrm{O}$ & $0-16,19$ \\
\hline 8 & Charca Salinera Chojon & 117 & $21^{\circ} 35^{\prime} 54.68^{\prime \prime} \mathrm{N}$ & $87^{\circ} 59^{\prime} 3.98^{\prime \prime} \mathrm{O}$ & $0-8,10-12,15$ \\
\hline 9 & Charca Salinera Cruz Grande & 3 & $21^{\circ} 36^{\prime} 0.95^{\prime \prime} \mathrm{N}$ & $87^{\circ} 59^{\prime} 25.00^{\prime \prime} \mathrm{O}$ & 5,14 \\
\hline 10 & Charca Salinera Miguel Torres & 2 & $21^{\circ} 35^{\prime} 46.92^{\prime \prime} \mathrm{N}$ & $88^{\circ} 0 ’ 9.16^{\prime \prime} \mathrm{O}$ & 4 \\
\hline 11 & Charca Salinera Poceb Xac & 3 & $21^{\circ} 35^{\prime} 54.95^{\prime \prime} \mathrm{N}$ & $87^{\circ} 59^{\prime} 57.01^{\prime \prime} \mathrm{O}$ & $5,8,10$ \\
\hline 12 & Charca Salinera Podridos & 6 & $20^{\circ} 52^{\prime} 54.93 ” \mathrm{~N}$ & $90^{\circ} 23^{\prime} 4.01^{\prime \prime} \mathrm{O}$ & 1,2 \\
\hline 13 & Charca Salinera Polkos & 41 & $21^{\circ} 35^{\prime} 59.72^{\prime \prime} \mathrm{N}$ & $87^{\circ} 58^{\prime} 35.39^{\prime \prime} \mathrm{O}$ & $2-5,7,9-11,13-15,18$ \\
\hline 14 & Charca Salinera Salines & 33 & $21^{\circ} 36^{\prime} 17.88^{\prime \prime} \mathrm{N}$ & $87^{\circ} 59^{\prime} 1.85 " \mathrm{O}$ & $1-5,7,9-10,14$ \\
\hline 15 & Charca Salinera San Fernando & 66 & $21^{\circ} 34^{\prime} 25.39^{\prime \prime} \mathrm{N}$ & $87^{\circ} 52^{\prime} 12.81^{\prime \prime} \mathrm{O}$ & $0-5,7-16$ \\
\hline 16 & Charca Salinera Via 3 & 2 & $21^{\circ} 35^{\prime} 35.54^{\prime \prime} \mathrm{N}$ & $87^{\circ} 57^{\prime} 1.21^{\prime \prime} \mathrm{O}$ & 3 \\
\hline 17 & Charca Salinera Vía Oriente & 3 & $21^{\circ} 35^{\prime} 33.18^{\prime \prime} \mathrm{N}$ & $87^{\circ} 55^{\prime} 28.87^{\prime \prime} \mathrm{O}$ & $2,5,11$ \\
\hline 18 & Charca Salinera La Esperanza & 627 & $21^{\circ} 35^{\prime} 44.03^{\prime \prime} \mathrm{N}$ & $88^{\circ} 0 ’ 49.85^{\prime \prime} \mathrm{O}$ & $0-1,3,5-12$ \\
\hline 19 & Charca San Crisanto & 4 & $21^{\circ} 20^{\prime} 24.44^{\prime \prime} \mathrm{N}$ & $89^{\circ} 10^{\prime} 19.72 ” \mathrm{O}$ & $0-1$ \\
\hline 20 & Chuburná & 2 & $21^{\circ} 13^{\prime} 59.38^{\prime \prime} \mathrm{N}$ & $89^{\circ} 49^{\prime} 0.35^{\prime \prime} \mathrm{O}$ & 11,12 \\
\hline 21 & Fraccionamiento Fox, San Felipe & 1 & $21^{\circ} 33^{\prime} 0.03^{\prime \prime} \mathrm{N}$ & $88^{\circ} 14^{\prime} 8.91^{\prime \prime} \mathrm{O}$ & 0 \\
\hline 22 & Humedal carretera Sisal-Hunucmá & 3 & $21^{\circ} 8,59.92 ” \mathrm{~N}$ & $90^{\circ} 1 ’ 0.07^{\prime \prime} \mathrm{O}$ & $0,2,18$ \\
\hline 23 & Humedal Progreso & 4 & $21^{\circ} 16^{\prime} 0.01^{\prime \prime} \mathrm{N}$ & $89^{\circ} 37^{\prime} 59.98^{\prime \prime} \mathrm{O}$ & $1,3-4,11$ \\
\hline 24 & Isla La Angostura & 2008 & $21^{\circ} 32^{\prime} 58.98^{\prime \prime} \mathrm{N}$ & $87^{\circ} 49^{\prime} 25.97^{\prime \prime} \mathrm{O}$ & $0-18$ \\
\hline 25 & Isla Pájaros, Celestún & 159 & $20^{\circ} 54^{\prime} 56.01^{\prime \prime} \mathrm{N}$ & $90^{\circ} 20^{\prime} 49.97^{\prime \prime} \mathrm{O}$ & $0-13$ \\
\hline 26 & Laguna Baas, Sisal & 1 & $21^{\circ} 7^{\prime} 59.98^{\prime \prime} \mathrm{N}$ & $90^{\circ} 6 ’ 42.02 ” \mathrm{O}$ & 15 \\
\hline 27 & Laguna cerca Puerto Abrigo Celestún & 4 & $20^{\circ} 51^{\prime} 27.99^{\prime \prime} \mathrm{N}$ & $90^{\circ} 22^{\prime} 59.99^{\prime \prime} \mathrm{O}$ & $0-1$ \\
\hline 28 & Laguna Champolak, Sisal & 8 & $21^{\circ} 7^{\prime} 0.07^{\prime \prime} \mathrm{N}$ & $90^{\circ} 9^{\prime} 0.01 ” \mathrm{O}$ & $1,2,12$ \\
\hline 29 & Laguna Nahular, Sisal & 1 & $21^{\circ} 7^{\prime} 59.99^{\prime \prime} \mathrm{N}$ & $90^{\circ} 7^{\prime} 0.01 ” \mathrm{O}$ & 2 \\
\hline 30 & Mina de Oro & 1 & $21^{\circ} 22^{\prime} 32.01^{\prime \prime} \mathrm{N}$ & $88^{\circ} 58^{\prime} 60.00^{\prime \prime} \mathrm{O}$ & 10 \\
\hline 31 & Mulsunik, Ría Lagartos & 10 & $21^{\circ} 35^{\prime} 6.98^{\prime \prime} \mathrm{N}$ & $87^{\circ} 56^{\prime} 41.98^{\prime \prime} \mathrm{O}$ & $0,1,7,9-10,12$ \\
\hline 32 & Parador Dzinitún & 2 & $20^{\circ} 51^{\prime} 18.97 ” \mathrm{~N}$ & $90^{\circ} 23^{\prime} 34.02 ” \mathrm{O}$ & 0 \\
\hline 33 & Pilares, Celestún & 57 & $20^{\circ} 52^{\prime} 59.99^{\prime \prime} \mathrm{N}$ & $90^{\circ} 22^{\prime} 0.01^{\prime \prime} \mathrm{O}$ & $1-4,10-11,14-15,18$ \\
\hline 34 & Charca Salinera Plaza de Armas, Celestún & 1 & $20^{\circ} 52^{\prime} 40.00^{\prime \prime} \mathrm{N}$ & $90^{\circ} 22^{\prime} 57.01 ” \mathrm{O}$ & 1 \\
\hline 35 & Punta Manolo, El Cuyo & 57 & $21^{\circ} 28^{\prime} 59.96^{\prime \prime} \mathrm{N}$ & $87^{\circ} 39^{\prime} 0.01^{\prime \prime} \mathrm{O}$ & $1-5,7,9-11,13-15,18$ \\
\hline 36 & Punta Mecoh & 1336 & $21^{\circ} 32^{\prime} 58.61^{\prime \prime} \mathrm{N}$ & $87^{\circ} 49^{\prime} 26.07^{\prime \prime} \mathrm{O}$ & $0-8,10-12,14-16,19$ \\
\hline 37 & Ria Natural frente Yoluk & 46 & $21^{\circ} 35^{\prime} 27.36^{\prime \prime} \mathrm{N}$ & $88^{\circ} 1 ’ 52.24^{\prime \prime} \mathrm{O}$ & $0-12,15-16$ \\
\hline 38 & Salamandra, Laguna Rosada & 13 & $21^{\circ} 19^{\prime} 37.83^{\prime \prime} \mathrm{N}$ & $89^{\circ} 23^{\prime} 0.11^{\prime \prime} \mathrm{O}$ & $1-3,8-9,12-13$ \\
\hline 39 & Xcambo & 15 & $21^{\circ} 18^{\prime} 59.46^{\prime \prime} \mathrm{N}$ & $89^{\circ} 19^{\prime} 59.84^{\prime \prime} \mathrm{O}$ & $3,7-10,12-13$ \\
\hline
\end{tabular}

Se incluyen las coordenadas geográficas por área y las edades de los flamencos detectados.

The geographical coordinates of each area, the total number of individuals and the age of flamingos detected are included. 


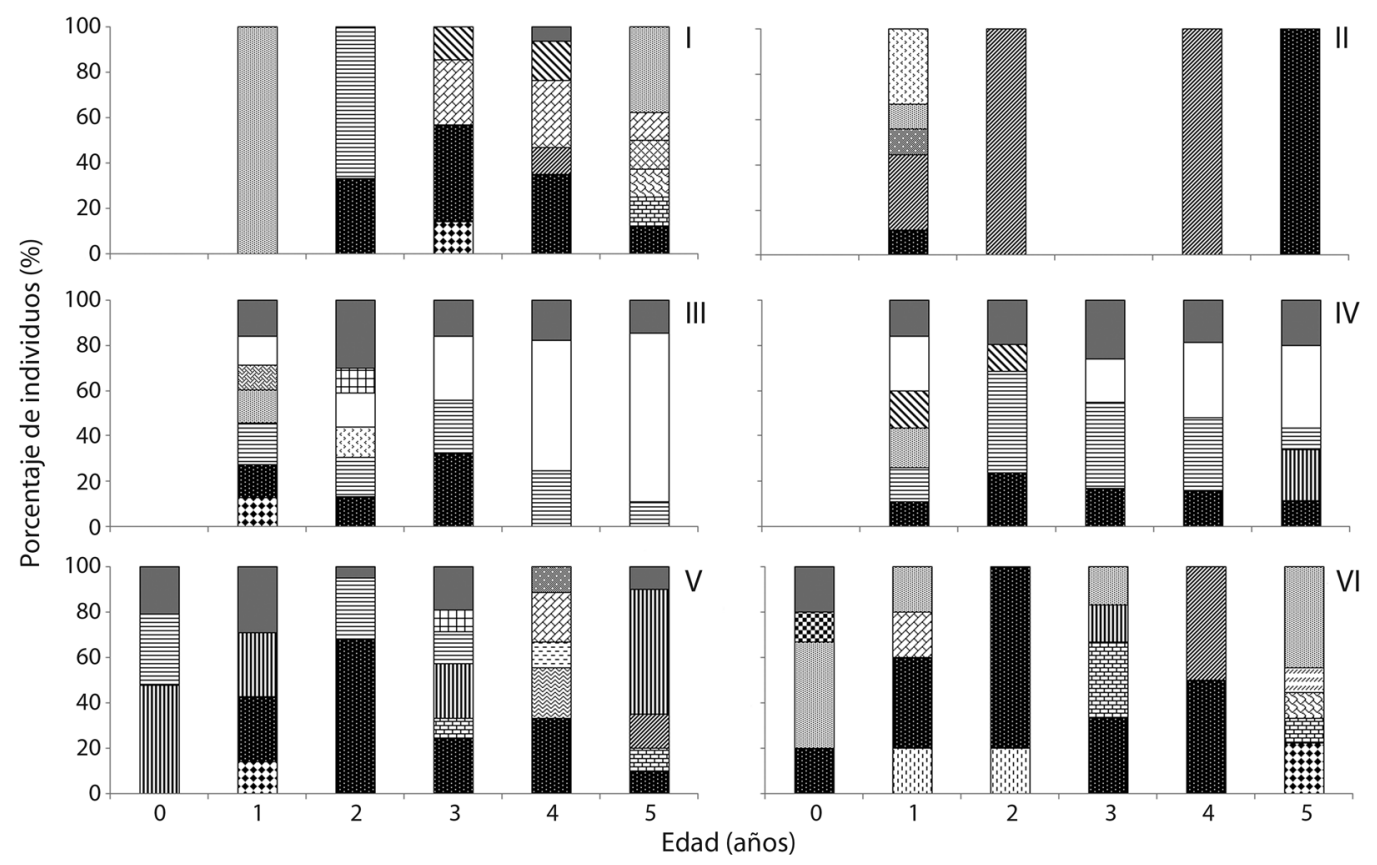

Fig. 2A. Áreas dentro de los humedales de Yucatán, México, con los mayores porcentajes en relación al número de individuos juveniles de flamenco caribeño (Phoenicopterus ruber) durante: I: Pre-reproductiva-nortes, II: Pre-Reproductivasecas, III: Reproductiva-secas, IV: Reproductiva-lluvias, V: Pos-reproductiva-lluvias y VI: Pos-reproductiva-nortes; durante el período 2010-2015. Otros: Incluye a todas las áreas donde se registró menos del $10 \%$ del total individuos por edad.

Fig. 2A. Areas within wetlands of Yucatan, Mexico, with the highest percentages of occupation by juveniles of Caribbean Flamingo (Phoenicopterus ruber) for: I: Pre-reproductive-north, II: Pre-Reproductive-dry, III: Reproductive-dry, IV: reproductive-rains, V: Post-reproductive-rains and VI: Post-reproductive-north; during the period 2010-2015. Other: Includes all areas where were registered less than $10 \%$ of all individuals by age.

Chel e Isla Pájaros, Celestún (Fig. 2B). Ya en Pre-R-S la mayoría de los adultos anillados registrados se movieron hacia las Charcas Salineras Chel y Chojon, por lo que se encontraron mayormente asociados en áreas cercanas a las de reproducción (Fig. 2B). Durante la etapa R-S y R-LL los sitios más importantes fueron Punta Mecoh e Isla La Angostura (Fig. 2B). Ya en Pos-R-LL los individuos de las diferentes edades se dispersaron más, y se encontraron más concentrados según la edad en varias de las Charcas Salineras o en áreas aledañas, así como en Xcambo; Mulsunik, Ría Lagartos o en Salamandra, Laguna Rosada (Fig. 2B).

\section{DISCUSIÓN}

El sistema de Charcas Salineras ISYSA (Industria Salinera de Yucatán, S.A. de C.V.) se destaca entre las más importantes para el flamenco caribeño en los humedales de Yucatán y aunque en estas se desarrollan actividades con fines industriales para la producción de sal, normalmente se ubican fuera de las áreas que históricamente han ocupado los flamencos de manera natural. Además, ISYSA ha tomado medidas para adecuar algunas de estas charcas para que no perturben a los flamencos que hacen uso de ellas. También es de destacar que, ISYSA ha financiado gran parte de las actividades de conservación del flamenco en Yucatán, y aunque es una industria con intereses económicos de por medio, se ha comprobado que el efecto de las salinas es mínimo, excepto por el impacto inicial durante la construcción de la infraestructura, que incluye destrucción del hábitat original. Luego de establecidas las salinas, el impacto en los alrededores es bajo 


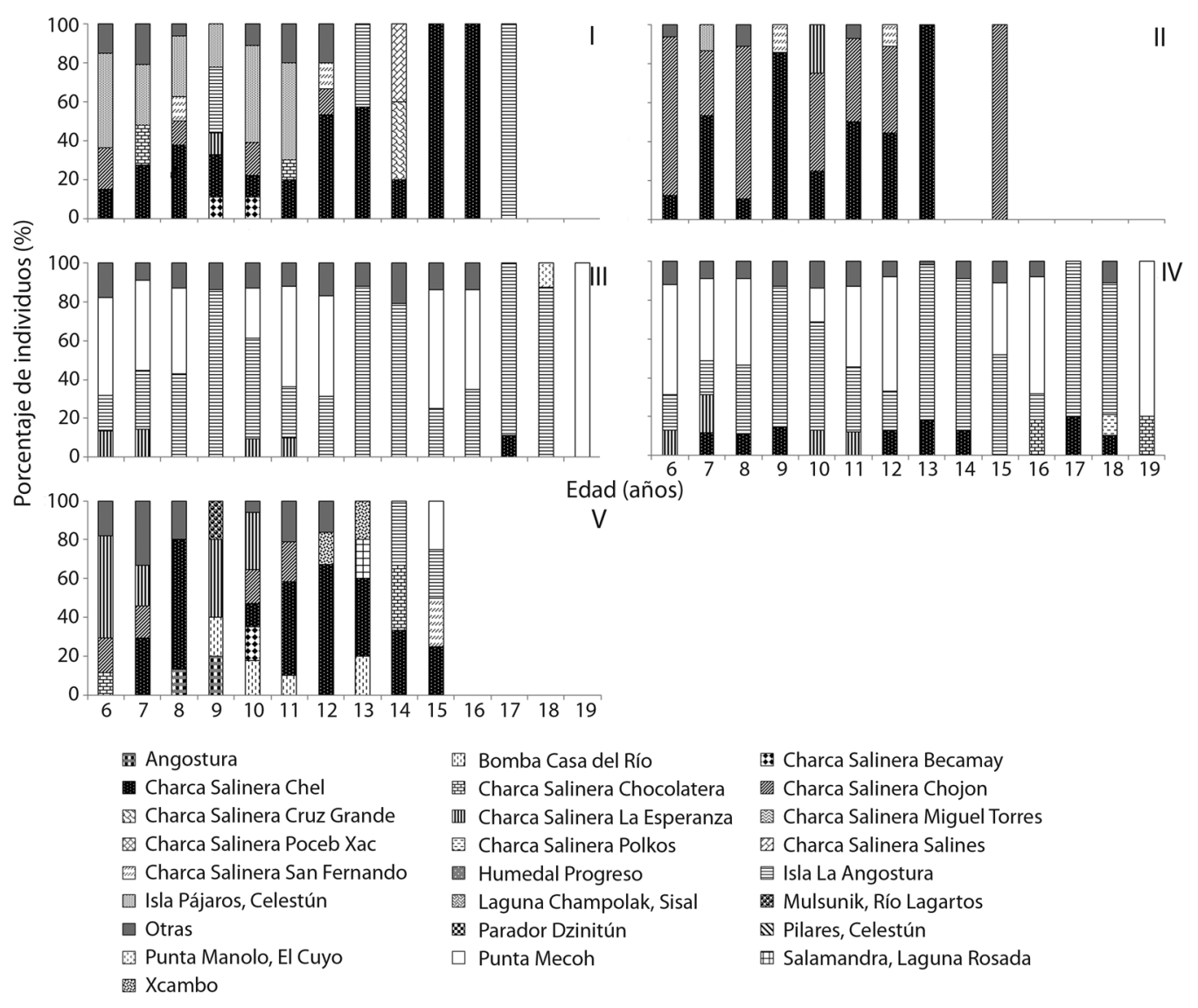

Fig. 2B. Áreas dentro de los humedales de Yucatán, México, con los mayores porcentajes en relación al número de individuos adultos de flamenco caribeño (Phoenicopterus ruber) durante: I: Pre-reproductiva-nortes, II: Pre-reproductivasecas, III: Reproductiva-secas, IV: Reproductiva-lluvias y V: Pos-reproductiva-lluvias; durante el período 2010-2015. Otros: Incluye a todas las áreas donde se registró menos del $10 \%$ del total individuos por edad.

Fig. 2B. Areas within wetlands of Yucatan, Mexico, with the highest percentages of occupation by adults of Caribbean Flamingo (Phoenicopterus ruber) for: I: Pre-reproductive-north, II: Pre-reproductive-dry, III: Reproductive-dry, IV: reproductive-rain and V: Post-reproductive-rain; during the period 2010-2015. Other: Includes all areas where were registered less than $10 \%$ of all individuals by age.

o resulta positivo para las aves, por la concentración de alimentos en las primeras fases del ciclo de obtención de la sal (Perez \& Hortas, 1991; Masero, 2003). Dentro de las charcas de ISYSA también hay cultivo de Artemia salina y cuando se libera el agua de estas a la Ría, para mantener los volúmenes, se incrementa el número de este crustáceo, el cual es uno de los que más consumen los flamencos durante la etapa de cría (Esté et al., 2014).

Además, el sistema de Charcas Salineras se encuentra aledaño a los principales sitios de reproducción de la especie y esta puede ser otra de las razones por las cuales los flamencos se asocian a las mismas en una proporción elevada. También debe tenerse en cuenta que los pollos de menos de cuatro meses, tienen una glándula especial con la que procesan la sal (Almansour, 2007), por ello pueden hacer uso de sitios con elevados niveles de salinidad. Después de los cuatro meses, comienzan a explorar otros sitios hasta que van alcanzando la madurez reproductiva, donde sus preferencias van cambiando nuevamente. 
CUADRO 2

Número total de individuos y avistamientos de flamenco caribeño (Phoenicopterus ruber), por edad, registrados durante el período 2010-2015 en diferentes áreas de los humedales de Yucatán, México

TABLE 2

Number of Caribbean Flamingo's (Phoenicopterus ruber) individuals and sightings, by age, registered during the period 2010-2015 in different areas of the Yucatan wetlands, Mexico wetlands

\begin{tabular}{cccc} 
Edad (años) & Total áreas & Total individuos & Total avistamientos \\
0 & 18 & $660(93 \pm 41,1-348)$ & $2354(407 \pm 131,1-1713)$ \\
1 & 22 & $197(12 \pm 10,1-38)$ & $266(16 \pm 12,1-56)$ \\
2 & 20 & $170(18 \pm 10,1-70)$ & $304(31 \pm 15,1-113)$ \\
3 & 19 & $278(29 \pm 17,1-101)$ & $631(62 \pm 33,1-181)$ \\
4 & 17 & $313(42 \pm 23,1-154)$ & $743(86 \pm 41,1-318)$ \\
5 & 18 & $201(26 \pm 13,1-111)$ & $491(55 \pm 27,1-219)$ \\
6 & 11 & $197(25 \pm 21,1-89)$ & $386(48 \pm 35,1-164)$ \\
7 & 17 & $293(34 \pm 21,1-126)$ & $699(75 \pm 41,1-267)$ \\
8 & 14 & $313(49 \pm 27,1-146)$ & $763(106 \pm 51,1-309)$ \\
9 & 17 & $308(61 \pm 21,1-254)$ & $775(147 \pm 46,1-608)$ \\
10 & 20 & $400(51 \pm 23,1-220)$ & $1064(130 \pm 53,1-549)$ \\
11 & 18 & $397(47 \pm 26,1-175)$ & $916(100 \pm 51,1-336)$ \\
12 & 17 & $305(46 \pm 21,1-180)$ & $704(95 \pm 41,1-363)$ \\
13 & 10 & $224(64 \pm 25,1-205)$ & $606(157 \pm 61,1-499)$ \\
14 & 10 & $258(67 \pm 29,1-216)$ & $684(168 \pm 68,1-542)$ \\
15 & 12 & $175(30 \pm 16,1-82)$ & $418(73 \pm 35,1-207)$ \\
16 & 7 & $66(15 \pm 11,1-41)$ & $124(23 \pm 18,1-63)$ \\
17 & 2 & $22(12 \pm 13,4-21)$ & $99(54 \pm 50,11-88)$ \\
18 & 6 & $18(6 \pm 4,1-16)$ & $44(12 \pm 7,1-32)$ \\
19 & 2 & $8(4 \pm 4,1-7)$ & $18(10 \pm 9,2-16)$ \\
\hline & & & \\
\hline
\end{tabular}

Estadísticos de posición y dispersión (media del número de individuos y de avistamientos por año y por área \pm desviación estándar, mínimo-máximo).

Statistical position and dispersion (average number of individuals and sightings by year by area \pm standard deviation, minimum-maximum).

Isla Pájaros en Celestún se destaca también como un sitio de gran importancia para la especie de manera general, lo cual ya se había documentado anteriormente pero principalmente fuera de la reproducción (Baldasarre \& Arengo, 2000; Gálvez et al., 2014). Aunque en este estudio se observó una mayor proporción de individuos juveniles a lo largo del año haciendo uso de esta área. En esta zona, el turismo basado en la observación de flamencos constituye un beneficio económico importante para las comunidades costeras. Sin embargo, a pesar de que existe un programa de sensibilización a los prestadores del servicio, y de haberse instaurado las normativas de no acercamiento de las lanchas a menos de $50 \mathrm{~m}$ de los bandos, así como establecer senderos acuáticos señalizados, aún existen problemas en este sentido. Los barcos turísticos de motor que se emplean perturban a los flamencos y reducen su tiempo diario de alimentación hasta en un 60 \% (Galicia \& Baldassare, 1997).

Del éxito que tengan los individuos más jóvenes de la población dependerá el futuro de la colonia a largo plazo y de ahí la importancia de que se logre el respeto a las normas establecidas y se continúe el programa de sensibilización y vigilancia. Además, se debe implementar un control a los prestadores de servicio y tener en cuenta su comportamiento 
para otorgar el permiso anual para operar. Todo esto basado en la importancia de mantener protegidas las áreas donde los flamencos se desarrollan y de emplear embarcaciones que los perturben en menor grado.

Entre las áreas más importantes durante la etapa reproductiva (secas y lluvias), tanto para adultos como para juveniles, se identificaron a Isla La Angostura y Punta Mecoh; lo cual era un resultado previsible ya que son actualmente los principales sitios de anidación reportados para la especie en la región (Rubio, 2010; Gálvez, Guerrero, \& Migoya, 2016). Durante la reproducción se forman colonias densas que reúnen a miles de individuos (Escalante, 2010), lo cual aumenta la probabilidad de poder detectar a aquellos que estén anillados. Además, las colonias reproductivas se localizan en áreas que están protegidas y monitoreadas, lo cual facilita también la detección. Aunque los juveniles no se reproducen, también se mueven a las áreas de reproducción, ya que apoyan a los adultos en actividades como la construcción de nido.

Sin embargo, aun cuando las áreas de reproducción estén protegidas, durante esta etapa las poblaciones pueden ser más sensibles a sufrir una declinación rápida, ya que se concentran en colonias grandes y en ecosistemas tan frágiles como los humedales, que pueden ser contaminados y fragmentados con facilidad (Faulkner, 2004). Para México en particular, los humedales se encuentran entre los ecosistemas más afectados por las actividades del hombre, tanto de manera directa como indirecta (Lara et al., 2008). El sistema acuífero de la Península de Yucatán está contaminado con medicamentos, pesticidas y otros productos químicos peligrosos que atentan contra la vida de los flamencos y todas las especies que conviven en las áreas de humedales (Escalante, 2010).

Fuera de la etapa reproductiva, los registros de individuos anillados se dificultan, ya que este tipo de asociaciones a gran escala que se dan durante la reproducción es menor, y los flamencos tienden a dispersarse más. Durante la etapa Pos-R-LL, aunque una parte considerable de los adultos se mantienen haciendo uso de las Charcas Salineras, otras áreas como Salamandra, Laguna Rosada y Xcambo resaltan como importantes. Ambas áreas se encuentran ubicadas dentro de la costa norte de Yucatán, la cual se ha reportado como una de las que alberga la mayor abundancia de flamencos en la región fuera de la etapa no reproductiva (Gálvez et al., 2014). Al igual que los adultos, en los juveniles se observa una tendencia a ocupar con mayor frecuencia las Charcas Salineras durante la etapa Pos-RLL y Pos-R-N, aunque durante la estación de nortes Isla Pájaros, Celestún también destaca como un área importante para ellos, lo cual concuerda con los resultados de Gálvez et al. (2014). Sin embargo durante las lluvias, aparte de las Charcas Salineras e Isla La Angostura, los sitios donde se registraron más juveniles fueron el Humedal Progreso y Salamandra, Laguna Rosada; lo cual nos indica que antes de moverse a Celestún se concentran en mayor número en estas áreas.

Aun cuando Salamandra, Laguna Rosada y Xcambo se encuentren dentro del Área Natural Protegida Reserva Estatal Ciénagas y Manglares de la Costa Norte de Yucatán, la situación de protección y vigilancia no es la misma que dentro de las Reservas de la Biosfera Celestún y Ría Lagartos. En las Reservas Estales, las actividades humanas no integradas al buen funcionamiento de los ecosistemas están menos supervisadas y por tanto, se generan hacia su interior problemas medioambientales más serios y más difíciles de controlar. Además, independientemente de las actividades antropogénicas, la ocurrencia de huracanes e incendios en esta zona afectan directamente a los ecosistemas y las diversas especies que los habitan.

La Laguna Champolak y Laguna Nahular, ambas en Sisal, y Chuburná; no sobresalieron entre las áreas más importantes, lo cual no coincide con los resultados obtenidos por Gálvez et al. (2014). Estos autores comprobaron que para el período Octubre 2011-Octubre 2012, en los humedales de Sisal y en Chuburná, se localiza gran parte de la población de flamencos de la Península de Yucatán fuera de las áreas de reproducción. Esta situación puede estar dada por el hecho de que algunas 
regiones son más fáciles de monitorear que otras y se obtiene mayor número de registros de individuos anillados en aquellas que son más accesibles, como es el caso de las áreas de reproducción en Ría Lagartos y otras como Celestún.

En áreas como la de los humedales de Sisal o Chuburná resulta más compleja la lectura de los anillos, porque es difícil acceder a las mismas y los puntos desde los cuales se pueden monitorear las colonias quedan algo alejados y no se pueden distinguir con precisión. Esta situación no es un problema cuando solo se realizan conteos para medir densidad o abundancia de la población. Esta situación se hace extensiva a muchas otras áreas dentro de los humedales de la Península de Yucatán, ya que de las 39 donde se lograron registrar flamencos anillados, solo a un número reducido se puede acceder con facilidad y se pueden realizar de manera más efectiva las lecturas de los anillos. Para varias áreas se cuenta con mayor información, gracias a los censos aéreos, en los cuales no es posible obtener información precisa sobre los individuos anillados.

Con estos resultados se pueden definir nuevas prioridades en relación a la conservación de la especie dentro de los humedales de Yucatán, particularmente prestándole más atención a las nuevas áreas que se perfilan como sitios de importancia para los flamencos. La información existente para el manejo y conservación de la especie estaba muy sesgada al solo tener en cuenta un grupo de investigaciones concentradas en un número reducido de áreas que históricamente habían sido definidas como las únicas importantes para los flamencos (e.g. Espino \& Baldassarre, 1989; Felicity \& Baldassarre, 1999; Herrera, Zaldivar, Ramírez, \& Alonso, 2000; Baldasarre \& Arengo, 2000). Este estudio apoya que los patrones de distribución de la especie en Yucatán son un poco más complejos, ya que la ocupación de las diferentes áreas está ligada directamente a las variaciones que se puedan dar durante las diferentes estaciones climáticas y no afectan de la misma manera a los individuos de los diferentes grupos etarios. Estos patrones ya se habían comenzado a describir en estudios más recientes (e. g. Gálvez et al., 2014, 2016).

Además, para el manejo de la especie debe tenerse en cuenta que las áreas que actualmente no son las de mayor importancia pueden serlo en un futuro. Los cambios de origen natural o antrópico en los hábitats que ocupa el flamenco caribeño provocan movimientos entre estaciones y etapas climáticas, que cambian por completo los patrones de distribución conocidos y hacen su ciclo de vida complejo y en constante variación. Aun cuando en la actualidad se lleven a cabo acciones de protección, rehabilitación y sensibilización en las colonias de reproducción, hay que mantener la vigilancia y establecer medidas en las áreas de crecimiento y en otras donde cumplen otras funciones biológicas importantes, para mantener una población residente estable en los humedales de la Península de Yucatán.

Se le debe dar seguimiento a los programas de educación ambiental existentes, para continuar incentivando un cambio en la actitud de las personas, principalmente en aquellas encargadas de las actividades turísticas en áreas de gran importancia como Celestún. De igual manera, se deben continuar la realización de campañas masivas en las cuales se divulgue toda la problemática alrededor de la especie, y a través de los medios de difusión masiva, alentar a las personas a tener más cuidado con la eliminación de sus desechos tanto sólidos como líquidos, para así contribuir con una disminución de la contaminación del sistema acuífero que alimenta los humedales donde habitan los flamencos. También se deben continuar con la búsqueda de fuentes de apoyo para la realización de estudios, que determinen y supervisen el estado de las poblaciones en aquellas áreas de difícil acceso, de las cuales aún se cuenta con muy poca información.

\section{AGRADECIMIENTOS}

Al personal de Niños y Crías A.C. por los datos facilitados para la elaboración de este estudio. A los patrocinadores del proyecto de conservación del flamenco en la Península 
de Yucatán: Fondo Mexicano para la Conservación de la Naturaleza A.C., Fundación Pedro y Elena Hernández A.C., International Flamingo Foundation, Texas A\&M Dallas Zoo, African Safari, parque Ecoarqueológico de Xcaret, Zoológicos de Mérida y Tizimín, Fundación BEPENSA y a la Industria Salinera de Yucatán S.A de C.V. A la Dirección de la Reserva de la Biosfera de Ría Lagartos, de la Comisión Nacional de Áreas Naturales Protegidas y la Procuraduría Federal de Protección al Ambiente. A los revisores del manuscrito por sus comentarios.

\section{RESUMEN}

El flamenco caribeño (Phoenicopterus ruber) en México se distribuye en todo el complejo de lagunas dentro de los humedales costeros de la Península de Yucatán y se ha observado una cierta tendencia a un uso diferencial de las áreas entre los juveniles y adultos en las diferentes etapas de su ciclo de vida, según las estaciones del año. El objetivo de este estudio fue determinar las áreas más importantes donde se distribuyen espacialmente los flamencos en los humedales de Yucatán, México y sus variaciones temporales según la edad y las diferentes etapas de su ciclo de vida. Se utilizaron registros de individuos anillados que se han avistado durante el período 2010-2015. Se identificaron las áreas con el mayor número de individuos según la estación y la temporada. Se registraron flamencos en 39 áreas diferentes, de 43 muestreadas. En general, los sitios con el mayor número de registros fueron: La Angostura y Punta Mecoh. Tanto para los juveniles como para los adultos, las áreas con mayores porcentajes de ocupación fueron diferentes por edades entre las diferentes etapas del ciclo de vida y las estaciones del año. Charca Salinera Chel e Isla La Angostura fueron áreas en las que se registraron flamencos anillados de casi todas las edades. Aunque se siguen destacando como zonas importantes para la especie, ya descritas con anterioridad (Celestún, Ría Lagartos), aparecen nuevos sitios que albergan un número considerable de la población en algún momento de su ciclo de vida (e. g. Humedal Progreso. Salamandra, Laguna Rosada; Xcambo). A estos nuevos sitios se les debe prestar más atención y se recomiendan medidas de seguridad y protección importantes para todos ellos. Es necesario buscar nuevas fuentes de apoyo para realizar estudios que determinen el estado de las poblaciones de flamencos en áreas de difícil acceso de las que se tiene muy poca información.

Palabras clave: adultos, especies amenazadas, flamenco caribeño, juveniles, Península de Yucatán, Phoenicopteridae.

\section{REFERENCIAS}

Almansour, M. I. (2007). Anatomy, histology and histochemistry of the salt glands of the greater flamingo Phoenicopterus rubber roseus (Aves, Phoenicopteridae). Saudi Journal of Biological Sciences, 14, 137-144.

Andrade, M. (2010). Transformación de los sistemas naturales por actividades antropogénicas. En R. Durán, \& M. Méndez (Eds.), Biodiversidad y desarrollo humano en Yucatán (pp. 316-319). México: CICY, PPD-FMAM, CONABIO, SEDUMA. Recuperado de http://www.seduma.yucatan.gob.mx/biodiversidadyucatan/03Parte2/Capitulo6/01 Transformacion sistemas_naturales.pdf

Baldasarre, G. A., \& Arengo, F. (2000). A Review of the Ecology and conservation of Caribbean Flamingos in Yucatán, Mexico. Waterbirds, 23, 70-79. DOI: $10.2307 / 1522149$

CONABIO. (2006). Mapa base del estado de Yucatán. Catálogo de metadatos geográficos. Comisión Nacional para el Conocimiento y Uso de la Biodiversidad. Recuperado de http://www.conabio. gob.mx/informacion/metadata/gis/yucaprgn.xml? $\mathrm{httpcache}=$ yes\&_xsl=/db/metadata/xsl/fgdc_html. $\mathrm{xsl} \&$ indent $=$ no

Cueto, V. R., Milesi, F. A., Sagario, M. C., López, J., \& Marone, L. (2011). Distribución geográfica y patrones de movimiento de la Monterita Canela (Poospiza ornata) y el Yal Carbonero (Phrygilus carbonarius) en Argentina. Ornitología Neotropical, 22, 483-494. Recuperado de https://sora.unm.edu/sites/default/ files/ON\%2022\%284\%29\%20483-494.pdf

Donázar, A., Travaini, A., \& Ceballos, O. (1999). Effects of sex-associated competitive asymmetries on foraging group structure and despotic distribution in Andean Condor. Behavioral Ecology and Sociobiology, 45, 55-65. Recuperado de http://www.jstor.org/ stable/4601576

Escalante, P. (2010). Ficha técnica de Phoenicopterus ruber. (Fichas sobre las especies de aves incluidas en Proyecto de Norma Oficial Mexicana PROY-NOM059-ECOL-2000, Parte 1, Bases de datos SNIBCONABIO, Proyecto No. W007.). D.F.: Instituto de Biología, UNAM.

Espino, R., \& Baldassarre, G. A. (1989). Numbers, migration chronology, and activity patterns of nonbreeding Caribbean Flamingos in Yucatan, Mexico. The Condor, 91, 592-597. DOI: 10.2307/1368109

Esté, E., Casler, C., Reyes, J., Nava, M., Carruyo, J., Flores, A., \& Pardo, C. (2014). Abundancia del flamenco caribeño (Phoenicopterus ruber) y su relación con las fuentes de alimento, en la ciénaga de los Olivitos, Venezuela. Boletín del Centro de Investigaciones Biológicas, 46(3), 211-332. 
Faulkner, S. (2004). Urbanization impacts on the structure and function of forested wetlands. Urban Ecosystems, 7, 89-106. DOI:10.1023/B:UECO.0000036269.56249.66

Felicity, A., \& Baldassarre, G. A. (1999). Resource variability and conservation of American Flamingos in coastal wetlands of Yucatán, Mexico. The Journal of Wildlife Management, 63(4), 1201-1212. DOI: $10.2307 / 3802838$

Galicia, E., \& Baldassarre, G. (1997). Effects of motorized tourboats on Caribbean Flamingos. Conservation Biology, 47, 298-302.

Gálvez, X., Gavaldón, A., Cruz, N., Guerrero, L., \& Marvi, E. (2014). Distribución, abundancia y composición por edades de los bandos de Flamenco (Phoenicopterus ruber) fuera de sus áreas de reproducción en los humedales de Yucatán, México. Revista Cubana de Ciencias Biológicas, 3(3), 52-59. Recuperado de http://www.rccb.uh.cu/index.php/RCCB/article/ view/277

Gálvez, X., Guerrero, L., \& Migoya, R. (2016). Evidencias físicas de la estructura metapoblacional en el flamenco caribeño (Phoenicopterus ruber ruber) a partir de avistamientos de individuos anillados. Revista Cubana de Ciencias Biológicas, 4(3), 93-98. Recuperado de http://www.rccb.uh.cu/index.php/RCCB/ article/view/382

García, E. (2004). Modificaciones al sistema de clasificación climática de Köppen. México: Instituto de Geografía-UNAM. Recuperado de http:/www.igeograf. unam.mx/sigg/utilidades/docs/pdfs/publicaciones/ geo_siglo21/serie_lib/modific_al_sis.pdf

Herrera, J. A., Zaldivar, A., Ramírez, J., \& Alonso, D. (2000). Habitat use of the American Flamingo (Phoenicopterus ruber ruber) in the Celestún Lagoon, Yucatan, Mexico. En F. A. Comin, J. A. Herrera, \& J. Ramírez (Eds.), Proceedings of the Limnology and Waterfowl, Monitoring, Modeling and Management Workshop (pp. 69-80). Mérida: Universidad Autónoma de Yucatán. Recuperado de https://www.researchgate.net/profile/Jorge_Silveira/ publication/268431387_HABITAT_USE_OF_THE_ AMERICAN_FLAMINGO Phoenicopterus_ruber ruber_IN_THE_CELESTUN_LAGOON_YUCATAN MEXICO/links/5540dc $\overline{7} \overline{1} 0 \mathrm{cf} 232222 \overline{7} 303 \mathrm{c} 82$. pdf? origin=publication_list

Lara, J. R., Arreola, J. A., Calderón, L. E., Camacho, V. F., de la Lanza, G., Escofet, A. M., Espejel, M. I., Guzmán, M., Ladah, L. B., López, M., Meling, E. A., Moreno, P., Reyes, H., Ríos, E., \& Zertuche, J. A. (2008). Los ecosistemas costeros, insulares y epicontinentales. En J. Soberón, G. Halffter, \& J. Llorente (Comps.), Capital natural de México I: Conocimiento actual de la biodiversidad (109-134). México: Comisión Nacional para el Conocimiento y Uso de la Biodiversidad. Recuperado de http:// www.biodiversidad.gob.mx/pais/pdf/CapNatMex/ Vol\%20I/I04_Losecosistemascos.pdf

Latta, S. C., \& Faaborg, J. (2002). Demographic and population responses of Cape May warblers wintering in multiple habitats. Ecology, 83, 2502-2515. DOI: $10.2307 / 3071810$

Marchetti, K. \& Price, T. (1989). Differences in the foraging of juvenile and adult birds: the importance of developmental constraints. Biological Reviews, 64(1), 51-70. DOI: 10.1111/j.1469-185X.1989.tb00638.x

Masero, J. A. (2003). Assessing alternative anthropogenic habitats for conserving waterbirds: salinas as buffer areas against the impact of natural habitat loss for shorebirds. Biodiversity and Conservation, 12(6), 1157-1173. DOI: 10.1023/A:1023021320448

Mendoza, L. F., Cortés, E., Sierra, C., \& Carmona, R. (2013). Avistamientos de flamencos (Phoenicopterus ruber) en la Reserva de la Biosfera Marismas Nacionales, Nayarit. Huitzil, 14(2), 84-86. Recuperado de http://www.scielo.org.mx/pdf/huitzil/v14n2/v14n2a2. pdf

Migoya, R. \& Tabasco, M. (2010). Programa integral de conservación del flamenco. En R. Durán \& M. Méndez (Eds.), Biodiversidad y desarrollo humano en Yucatán (pp. 432-434). México: CICY, PPD-FMAM, CONABIO, SEDUMA. Recuperado de http://www. cicy.mx/Documentos/CICY/Sitios/Biodiversidad/ pdfs/Cap $8 / 09 \% 20$ Programa $\% 20$ integral $\% 20 \mathrm{de} \% 20$ flamenco.pdf

Pérez, A., \& Hortas, F. (1991). Information about the habitat use of salines and fish ponds by wintering waders in Cadiz Bay, Southwest Spain. Wader Study Group Bulletin, 66, 48-53. Recuperado de https:// sora.unm.edu/sites/default/files/journals/iwsgb/n066/ p00048-p00053.pdf

Pérez, J., \& Telleria, L. (2002). Migratory and sedentary blackcaps in sympatric non-breeding grounds: implications for the evolution of avian migration. Journal of Animal Ecology, 71, 211-224. DOI: 10.1046/j.1365-2656.2002.00590.x

Plasencia, A. H., Escalona, G., \& Esparza, L. G. (2014). Interaction of landscape variables on the potential geographical distribution of parrots in the Yucatan Peninsula, Mexico. Animal Biodiversity and Conservation, 37.2, 191-203. Recuperado de 
http://abc.museucienciesjournals.cat/files/ABC_372_pp_191-203.pdf

Rubio, R. (2010). El flamenco rosa del Caribe y su conservación. En J. Carabias, J. Sarukhán, J. de la Maza, \& C. Galindo (Coords.), Patrimonio natural de México. Cien casos de éxito (pp. 62-63). México: Comisión Nacional para el Conocimiento y Uso de la Biodiversidad. Recuperado de http://www.biodiversidad.gob. $\mathrm{mx} /$ pais/cien_casos/pdf/cap23.pdf
Schmitz, R. A., Alonso, A. Cook, R. S., \& Bald, G. A. (1990). Lead poisoning of Caribbean Flamingos in Yucatan, Mexico. Wildlife Society Bulletin, 18, 399-404. Recuperado de http://www.jstor.org/ stable/3782738

Thomas, N. J., Hunter, D. B., \& Atkinson, C. T. (Eds.). (2008). Infectious diseases of wild birds. Oxford: Blackwell Publishing. DOI: 10.1002/9780470344668.ch6 